Rev. Bras. Saúde Prod. Anim., Salvador, v.17, n.2, p.214-221 abr./jun., 2016 http://www.rbspa.ufba.br

\title{
Gravidade específica de ovos de matrizes pesadas com diferentes idades no rendimento de incubação e no peso dos pintos pós-eclosão
}

\author{
Specific gravity of eggs of female broiler breeders in the yield of incubation and chick \\ weight post-hatch
}

\author{
SILVA, Mônica Calixto da ${ }^{1}$; NOLETO, Raiana Almeida ${ }^{2}$; VAZ, Roberta Gomes \\ Marçal Vieira ${ }^{1}$; COSTA, Eduardo Santos ${ }^{3}$; SOUSA, Luciano Fernandes ${ }^{1}$; \\ RODRIGUES, Kênia Ferreira ${ }^{1}$; SOUSA, Joana Patrícia Lira de ${ }^{1}$; CRUZ, Lília Alves ${ }^{1}$; \\ FONSECA, Flávia Luzia Rodrigues ${ }^{1}$
}

\footnotetext{
${ }^{1}$ Universidade Federal do Tocantins, Escola de Medicina Veterinária e Zootecnia, Departamento de Produção Animal, Araguaína, Tocantins, Brasil

${ }^{2}$ Universidade Federal de Goiás, Escola de Medicina Veterinária e Zootecnia, Departamento de Produção Animal, Goiânia, Goiás, Brasil.

${ }^{3}$ ASA Alimentos, Brasília, Distrito Federal, Brasil.

*Endereço para correspondência: monicalixto18@yahoo.com.br
}

\section{RESUMO}

Objetivou-se neste trabalho avaliar o efeito da gravidade específica de ovos de matrizes pesadas com diferentes idades no rendimento de incubação e no peso dos pintos pós-eclosão. Foram utilizados 3.072 ovos oriundos de dois lotes de matrizes pesadas, linhagem Cobb $500^{\circledR}$. Os ovos foram distribuídos em delineamento experimental inteiramente casualizado, em esquema fatorial $2 \times 4$, com duas idades de matrizes (53 e 64 semanas) e quatro diferentes densidades $(1.070,1.075,1.080$, $1.085)$, totalizando oito tratamentos e quatro repetições (com 96 ovos por unidade experimental). As variáveis avaliadas foram o peso do ovo (g), a taxa de eclosão (\%), a taxa de fertilidade $(\%)$, a perda de peso dos ovos durante o período de incubação (\%) e a relação do peso dos pintos/peso dos ovos (\%). Os resultados foram submetidos à análise de variância, sendo que o efeito das densidades foi avaliado por meio de regressão polinomial e o efeito da idade das matrizes pelo teste $\mathrm{F}$, ao nível de $5 \%$ de probabilidade. As perdas de peso dos ovos, exceto para as densidades de $1.075 \mathrm{e}$ 1.085 , oriundos de matrizes com 53 e 64 semanas de idade, respectivamente, apresentaram-se dentro dos valores recomendados na literatura. $\mathrm{O}$ peso do pinto ao nascer foi proporcional ao peso do ovo, representando em média $68 \%$ e sem diferenças entre a idade das matrizes $\mathrm{e}$ as diferentes densidades. A idade da matriz exerceu influência no rendimento de incubação, sendo as maiores taxas de fertilidade e eclosão total $(p<0,05)$ obtida pelos ovos oriundos de matrizes com 53 semanas de idade.

Palavras-chave: densidade de ovos, fertilidade, ovos incubáveis, taxa de eclosão

\section{SUMMARY}

The objective of this work was to evaluate the effect of specific gravity of eggs from broiler breeders of different ages in the yield of incubation and the weight of post-hatch chicks. Were used 3.072 eggs from two batches of broiler breeders, Cobb - $500^{\circledR}$. The eggs were distributed a completely randomized design in a $2 \times 4$ factorial design with two broiler breeder ages (53 and 64 weeks) and four different egg densities $(1.070,1.075,1.080,1.085)$, totaling eight treatments and four replications (with 96 eggs per experimental unit). The evaluated variables were the egg weight $(\mathrm{g})$, the hatching rate $(\%)$, the fertility rate $(\%)$, the weight loss of eggs during the incubation period $(\%)$ and the ratio of the weight of the chicks/egg weight (\%). The results were submitted to analysis of 
variance, being that the effect of density was assessed by polynomial regression and the age effect of breeders by $\mathrm{F}$ test at $5 \%$ probability. Weight losses of eggs, except for the densities of 1,075 and 1,085, from breeders with 53 and 64 weeks of age, respectively, were within the values recommended in the literature. The chick weight was proportional to the weight of the egg, representing on average $68 \%$ and no differences between age and density of broiler breeders. The age of the breeders exerted influence on the yield of incubation, and the best results $(p<0.05)$ obtained by eggs from breeders with 53 weeks of age.

Keywords: density, hatching eggs, hatching rate, incubation

\section{INTRODUÇÃO}

A determinação da gravidade específica é um método indireto e eficaz para avaliar a qualidade da casca de ovos incubáveis. Além disso, vem sendo utilizado para determinar sua relação com fatores produtivos como: taxa de fertilidade, eclosão total, percentagem de perda de peso dos ovos durante o período de incubação, qualidade dos pintos de um dia e no seu desempenho a idade ao abate (INGRAM et al., 2008; MOYLE et al., 2008).

A idade da matriz é um dos principais fatores que interfere na qualidade da casca do ovo. Aves mais velhas produzem ovos mais pesados, entretanto, com menor espessura de casca e maior número de poros (CARVALHO et al., 2007), essa condição pode influenciar de forma negativa nos índices de eclosão e na qualidade de pintos de um dia (INGRAM et al., 2008).

Vale ressaltar a importância da relação entre o peso do ovo e a gravidade específica, em que o peso do ovo aumenta ao passo que a gravidade específica diminui com a idade das reprodutoras (ÁVILA et al., 2005).
Em estudo realizado por Carvalho et al. (2007) foi observado que ovos de poedeiras jovens, com 29 semanas de idade, apresentaram maior gravidade específica (1081) em relação aos ovos de poedeiras com 60 e 69 semanas de idade, com gravidade específica de 1075 e 1074, respectivamente. No entanto, o peso do ovo $(56,02 \mathrm{~g})$ e a percentagem de gema $(24,69 \%)$ foram inferiores para as matrizes com 29 semanas de idade, indicando que com o aumento da idade, as aves tendem a aumentar o tamanho dos ovos.

Com base no exposto, objetivou-se neste trabalho avaliar o efeito da gravidade específica de ovos de matrizes pesadas, no rendimento de incubação e no peso dos pintos póseclosão.

\section{MATERIAL E MÉTODOS}

O experimento foi conduzido no incubatório industrial, localizado em Brasília - DF, no período de 03 a 25 de setembro de 2010. Foram utilizados 3.072 ovos incubáveis oriundos de dois lotes de matrizes pesadas, da linhagem Cobb - $500^{\circledR}$, produzidos e coletados no mesmo dia.

A gravidade específica foi determinada pela imersão sequencial dos ovos em baldes contendo diferentes soluções salinas $(\mathrm{NaCl})$ de acordo com a metodologia proposta por Castelló et al. (1989), com densidades variando entre 1.070 e 1.085 e intervalos de 0,005, calibrados com densímetro da marca Incotem $^{\circledR}$. Em seguida, os ovos foram colocados em bandejas apropriadas para incubação, com capacidade para 96 ovos cada uma, devidamente identificadas por tratamentos e repetições para, posteriormente, serem pesadas individualmente. 
Rev. Bras. Saúde Prod. Anim., Salvador, v.17, n.2, p.214-221 abr./jun., 2016 http://www.rbspa.ufba.br

O delineamento experimental foi inteiramente ao acaso em esquema fatorial $2 \times 4$, com duas idades de matrizes (53 e 64 semanas de idade) e quatro diferentes densidades (1.070, $1.075,1.080,1.085)$, totalizando oito tratamentos e quatro repetições. Assim, cada bandeja com 96 ovos foi considerada uma repetição. Após pesados os ovos com peso médio de $69,63 \pm 0,79$ g para matrizes com 53 semanas e $69,19 \pm 0,61 \mathrm{~g}$ para matrizes com 62 semanas, ficaram estocados por um período de 24 horas na sala de armazenamento, com temperatura e umidade relativa do ar média de $21 \pm$ $0,4^{\circ} \mathrm{C}$ e $72 \pm 0,7 \%$, respectivamente. Após isso, os ovos foram pré-aquecidos a uma temperatura de $28^{\circ} \mathrm{C}$, durante 8 horas, com $62 \%$ de umidade relativa do ar. Em seguida, foram colocados em incubadora de estágio múltiplo, com temperatura e umidade relativa do ar média de $37,5 \pm 0,3^{\circ} \mathrm{C}$ e $55 \pm 0,4 \%$, respectivamente, com renovação constante de ar e viragem de $45^{\circ}$ para a direita e esquerda, de hora em hora.

Aos 19 dias de incubação foram feitas as transferências dos ovos da incubadora para o nascedouro, com temperatura e umidade relativa do ar média de $36,5 \pm$ $0,5^{\circ} \mathrm{C}$ e $54 \pm 0,7 \%$, respectivamente. Antes de serem colocadas nos nascedouros todas as bandejas foram pesadas, individualmente, para verificação da perda de umidade durante o período de incubação. Logo após o nascimento foram realizadas as pesagens e contagens dos pintos, para contabilizar o peso ao nascimento e a taxa de eclosão total, respectivamente.

As variáveis analisadas foram o peso inicial do ovo (g), peso do pinto na eclosão (g), relação do peso do pinto e peso do ovo (\%), perda de peso dos ovos durante o período de incubação $(\%)$, taxa de fertilidade (\%) e de eclosão total (\%).
Os índices percentuais de eclosão e fertilidade foram calculados segundo as equações propostas por Rosa \& Avila (2000): $\mathrm{E}=(\mathrm{TPN} / \mathrm{TOI}) \times 100 ; \mathrm{F}=$ (TOF/TOI) x 100. Em que: E, eclosão; TPN, total de pintos nascidos; TOI, total de ovos incubados; TOF, total de ovos férteis; F, fertilidade.

Os dados foram submetidos à análise variância pelo programa estatístico SISVAR (FERREIRA, 2010) sendo as densidades avaliadas por análises de regressão polinomial, testados os coeficientes lineares e quadráticos e as idades pelo teste $\mathrm{F}$, ao nível de $5 \%$ de probabilidade de erro tipo I.

\section{RESULTADOS E DISCUSSÃO}

Observou-se que ovos de matrizes mais novas, apresentaram maiores taxas de fertilidade quando comparados aos ovos das matrizes mais velhas, independentemente da gravidade específica $(p<0,05) \quad($ Tabela 1). Considerando a análise de regressão, verificou-se efeito quadrático para matrizes com 53 e 64 semanas de idade, com ponto máximo para ovos com gravidade específica de 1.079 e 1.078 , equivalendo a $90,23 \%$ e $78,72 \%$ de taxa de fertilidade, respectivamente.

Resultados divergentes foram encontrados por Roque \& Soares (1994), que ao trabalharem com dois grupos de ovos com gravidade específica maior que 1.080 (ovos com casca grossa) e menor que 1.080 (ovos com casca fina) $\mathrm{e}$ diferentes idades de matrizes $(27,31,40$, 44 e 55 semanas de idade), observaram maiores taxas de fertilidade em ovos com gravidade específica maior que 1.080 (96,5; 94,3\%), respectivamente. Ainda de acordo com os autores, o maior índice de fertilidade dos ovos com maior gravidade 
Rev. Bras. Saúde Prod. Anim., Salvador, v.17, n.2, p.214-221 abr./jun., 2016 http://www.rbspa.ufba.br ISSN 15199940

específica está associado com menor mortalidade embrionária.

De acordo com Fávero (2010) à medida que a ave envelhece, pode ocorrer uma diminuição no interesse pela cópula, o que possivelmente favoreceu para que as matrizes de 53 semanas de idade apresentassem melhores índices de fertilidade quando comparadas as matrizes de 64 semanas de idade.
$\mathrm{Na}$ comparação das médias da taxa de eclosão total (Tabela 2), nota-se que as matrizes com 53 semanas de idade tiveram melhores índices de eclosão total, quando comparados as matrizes com 64 semanas $(p<0,05)$.

$\mathrm{O}$ que resultou em efeito quadrático com ponto máximo para gravidade específica dos ovos de 1.078 e 1.080, correspondendo a uma taxa de eclosão de $83,56 \%$ e $62,5 \%$, respectivamente.

Tabela 1. Taxa de fertilidade (\%) dos ovos de matrizes pesadas de acordo com a idade e as diferentes densidades

\begin{tabular}{|c|c|c|c|c|c|c|c|c|}
\hline \multirow{2}{*}{$\begin{array}{l}\text { Idade } \\
\text { (semanas) }\end{array}$} & \multicolumn{4}{|c|}{ Densidades $\left(\mathrm{kg} / \mathrm{m}^{3}\right)$} & \multirow{2}{*}{ Médias } & \multirow{2}{*}{ Equação } & \multirow{2}{*}{$\mathrm{R}^{2}$} & \multirow{2}{*}{$\begin{array}{l}\text { CV } \\
(\%)\end{array}$} \\
\hline & 1070 & 1075 & 1080 & 1085 & & & & \\
\hline 53 & 84,25 & 89,00 & 91,75 & 88,75 & $88,44^{\mathrm{A}}$ & $\begin{array}{c}\hat{Y}=-90237,31+ \\
167,3375 x-0,0775 x^{2}\end{array}$ & 97,57 & \\
\hline 64 & 72,00 & 80,25 & 76,00 & 75,25 & $75,86^{\mathrm{B}}$ & $\begin{array}{l}\hat{Y}=-104530,40+ \\
194,06 x-0,090 x^{2}\end{array}$ & 62,97 & 9,72 \\
\hline Médias & 78,13 & 84,63 & 83,87 & 82,00 & 82,17 & $\begin{array}{c}\hat{Y}=-97383,85 *+ \\
180,70 * x-0,084 * x^{2}\end{array}$ & 92,59 & \\
\hline
\end{tabular}

*Significativo a $5 \%$ de probabilidade de erro; Médias com letras distintas na mesma coluna diferem significativamente a $5 \%$ de probabilidade de erro pelo teste de $\mathrm{F}$.

Tabela 2. Taxa de eclosão total (\%) dos ovos de matrizes pesadas de acordo com a idade e as diferentes densidades

\begin{tabular}{lcccccccc}
\hline $\begin{array}{l}\text { Idade } \\
\text { (semanas) }\end{array}$ & \multicolumn{9}{c}{ Densidades $\left(\mathrm{kg} / \mathrm{m}^{3}\right)$} & Média & Equação & $\mathrm{R}^{2}$ & $\begin{array}{l}\mathrm{CV} \\
(\%)\end{array}$ \\
\cline { 2 - 5 } 53 & 1070 & 1075 & 1080 & 1085 & $\mathrm{~s}$ & $\hat{\mathrm{Y}=-215133,75+}$ & 95,48 & \\
64 & 70,50 & 79,75 & 84,75 & 75,50 & $77,63^{\mathrm{A}}$ & $\begin{array}{c}399,075 \mathrm{x}-0,185 \mathrm{x}^{2} \\
\hat{\mathrm{Y}}=-122487,50+\end{array}$ & 78,39 & 9,72 \\
& 53,25 & 65,50 & 62,50 & 64,25 & $61,38^{\mathrm{B}}$ & $\begin{array}{c}226,875 \mathrm{x}-0,105 \mathrm{x}^{2} \\
\hat{\mathrm{Y}}=-168810,625^{*}+\end{array}$ & 98.53 & \\
Médias & 61,88 & 72,63 & 73,63 & 69,88 & & $312,975^{*} \mathrm{x}-0,145^{*} \mathrm{x}^{2}$ & 98.53 & \\
\hline
\end{tabular}

* Significativo a $5 \%$ de probabilidade de erro; Médias com letras distintas na mesma coluna diferem significativamente a $5 \%$ de probabilidade de erro pelo teste de $\mathrm{F}$.

As taxas de eclosão referentes às densidades de $1075(79,75 \%)$ e 1085 $(75,50 \%)$ também para as matrizes de 53 semanas de idade, estão de acordo com os valores encontrados por Barbosa et al. (2008), que obtiveram taxas de eclosão de $76,33 \%$ em ovos de matrizes com 56 semanas de idade e incubados com umidade relativa do ar de $56 \%$. Porém, deve-se enfatizar que no estudo realizado por estes autores, não foram avaliados a gravidade específica dos ovos, diferentemente da proposta do presente estudo, que foi avaliar os 
Rev. Bras. Saúde Prod. Anim., Salvador, v.17, n.2, p.214-221 abr./jun., 2016 http://www.rbspa.ufba.br ISSN 15199940

efeitos da idade da matriz na qualidade da casca dos ovos.

$\mathrm{Na}$ análise da Tabela 3 , verifica-se que as densidades interferiram significativamente $(p>0,05)$ nos valores de perda de peso dos ovos (\%) durante o período de incubação, apresentando efeito linear negativo e quadrático para as matrizes com 53 e 64 semanas de idade, respectivamente.

Os ovos provenientes das densidades de 1.075 e 1.085 , das matrizes com 53 e 64 semanas de idade, respectivamente, não alcançaram os valores percentuais para perda de peso recomendados por Rosa et al. (2002), em que a taxa de eclosão e a eclodibilidade foram otimizadas com taxa de perda de peso de ovos de $10,3 \%$, onde as taxas de eclosão para os ovos de matrizes com 53 e 63 semanas de idade foram de 85,2 e $82,8 \%$, respectivamente, valores superiores as taxas do presente trabalho de $77,63 \%$ para ovos de matrizes com 53 semanas e $61,38 \%$ para os ovos de matrizes com 64 semanas.

Tabela 3. Percentagem de perda de peso dos ovos aos 19 dias de incubação de acordo com a idade da matriz e as densidades

\begin{tabular}{|c|c|c|c|c|c|c|c|c|}
\hline \multirow{2}{*}{$\begin{array}{l}\text { Idade } \\
\text { (semanas) }\end{array}$} & \multicolumn{4}{|c|}{ Densidades $\left(\mathrm{kg} / \mathrm{m}^{3}\right)$} & \multirow{2}{*}{ Médias } & \multirow{2}{*}{ Equação } & \multirow{2}{*}{$\mathrm{R}^{2}$} & \multirow{2}{*}{$\begin{array}{l}\text { CV } \\
(\%)\end{array}$} \\
\hline & 1070 & 1075 & 1080 & 1085 & & & & \\
\hline 53 & 13,25 & 10,75 & 10,50 & 7,75 & 10,50 & $\hat{Y}=371,53^{*}-0,34 * x$ & 92,46 & \\
\hline 64 & 12,50 & 9,25 & 10,00 & 10,25 & 10,56 & $\begin{array}{c}\hat{\mathrm{Y}}=40773,93 *- \\
75,55^{*} \mathrm{x}+0,04 * \mathrm{x}^{2}\end{array}$ & 82,77 & 12,22 \\
\hline Médias & 12,87 & 10,00 & 10,25 & 9,00 & & $\hat{\mathrm{Y}}=255-0,23 \mathrm{x}$ & 78,90 & \\
\hline
\end{tabular}

*Significativo a $5 \%$ de probabilidade de erro; Médias na mesma coluna não diferem significativamente a $5 \%$ de probabilidade de erro pelo teste de F.

Os resultados encontrados neste trabalho assemelham aos descritos por Santos et al. (2009), onde as reprodutoras pesadas apresentaram melhor qualidade das barreiras contra evaporação e difusão de água dos ovos para o meio ambiente externo. À medida que aumenta a idade da ave, a qualidade de casca das poedeiras diminui consideravelmente, o que implica também em uma maior facilidade de contaminação dos ovos.

Ovos provenientes de aves mais velhas aumentam a espessura de casca o que leva ao excessivo número de poros afetando a troca gasosa (condutância à água), elevando a mortalidade precoce, resultante de toxidade à $\mathrm{CO}_{2}$ ou insuficiente perda de água (PEEBLES \& BRAKE, 1985).

Resultados contrastantes foram encontrados por Tanure et al. (2009) que observaram que ovos produzidos por matrizes de 57 semanas de idade, perderam mais peso em relação as matrizes com 32 semanas de idade. Tal fato pode ser explicado pela menor espessura da casca dos ovos de matrizes mais velhas, que promove maior perda de água durante o período de incubação, quando comparada com ovos de matrizes jovens.

$\mathrm{O}$ peso médio dos ovos $(\mathrm{g})$, o peso médio dos pintos $(\mathrm{g})$ assim como, os percentuais das relações entre o peso médio dos pintos/peso médio dos ovos (\%) apresentados na Tabela 4, são semelhantes aos valores encontrados por Almeida et al. (2006) \& Barbosa et al. (2008).

De acordo com Ávila et al. (2005) existe uma relação entre o peso do ovo e a gravidade específica, em que o peso 
Rev. Bras. Saúde Prod. Anim., Salvador, v.17, n.2, p.214-221 abr./jun., 2016 http://www.rbspa.ufba.br ISSN 15199940

do ovo aumenta e a gravidade específica diminuiu com o aumento da idade das aves. Entretanto, esse comportamento não foi observado no presente estudo, indicando que as diferenças entre os pesos médios dos ovos das matrizes de $53(69,19 \mathrm{~g})$ e $64(69,63 \mathrm{~g})$ semanas não influenciaram essa variável.

Tabela 4. Peso médio do ovo, peso médio do pinto ao nascimento e a relação do peso médio do ovo/peso

\begin{tabular}{|c|c|c|c|c|c|c|c|c|}
\hline \multirow{2}{*}{$\begin{array}{l}\text { Idade } \\
\text { (semanas) }\end{array}$} & \multicolumn{4}{|c|}{ Densidades $\left(\mathrm{kg} / \mathrm{m}^{3}\right)$} & \multirow{2}{*}{ Médias } & \multirow{2}{*}{ Equação } & \multirow{2}{*}{$\mathrm{R}^{2}$} & \multirow{2}{*}{$\begin{array}{l}\text { CV } \\
(\%) \\
\end{array}$} \\
\hline & 1070 & 1075 & 1080 & 1085 & & & & \\
\hline \multicolumn{9}{|c|}{ Peso dos ovos } \\
\hline 53 & 70,50 & 69,75 & 69,50 & 68,75 & 69,19 & $\hat{\mathrm{Y}}=188,15-0,11 \mathrm{x}$ & 96,80 & \\
\hline 64 & 69,25 & 69,50 & 69,00 & 69,00 & 69,63 & Ns & - & 1,22 \\
\hline Médias & 69,88 & 69,63 & 69,25 & 68,88 & & $\hat{Y}=142,14-0,07 x$ & 99,18 & \\
\hline \multicolumn{9}{|c|}{ Peso dos pintos } \\
\hline 53 & 46,75 & 46,50 & 46,00 & 46,50 & 46,44 & Ns & - & \\
\hline 64 & 46,75 & 46,50 & 46,25 & 46,75 & 46,56 & Ns & - & 1,64 \\
\hline Médias & 46,75 & 46,50 & 46,13 & 46,63 & & Ns & - & \\
\hline \multicolumn{9}{|c|}{ Relação do peso dos pintos/peso dos ovos } \\
\hline 53 & 66,31 & 66,66 & 66,19 & 67,64 & 66,70 & Ns & - & \\
\hline 64 & 67,51 & 66,91 & 67,03 & 67,75 & 67,30 & Ns & - & 1,43 \\
\hline Médias & 66,91 & 66,79 & 66,61 & 67,00 & & Ns & - & \\
\hline
\end{tabular}

*Significativo a $5 \%$ de probabilidade de erro; Médias com letras distintas na mesma coluna diferem significativamente a $5 \%$ de probabilidade de erro pelo teste de $\mathrm{F}$.

Em concordância com os dados encontrados por Tanure et al. (2009) \& Rocha et al. (2008), os resultados deste trabalho mostraram que o peso do pinto ao nascer foi proporcional ao peso do ovo, representando em média $67 \%$ e sem diferenças entre as idades das matrizes e as densidades. O que justifica a semelhança entre os pesos médios dos pintos ao nascer nas diferentes idades de matrizes, pois, os pesos médios dos ovos para matrizes com 53 semanas $(69,63 \mathrm{~g})$ e 62 semanas $(69,19 \mathrm{~g})$ eram praticamente iguais.

De acordo com os resultados obtidos no presente trabalho conclui-se, que a gravidade específica interfere no rendimento de incubação, apresentando um ponto máximo de gravidade específica de 1.079 e 1.078 para taxa de fertilidade e eclosão total, respectivamente.

\section{AGRADECIMENTOS}

Os autores expressam agradecimentos a Empresa Asa Alimentos LTDA pela doação dos ovos e das instalações para realização deste trabalho.

\section{REFERÊNCIAS}

ALMEIDA, J.G.; DAHLKE, F.; MAIORKA, A.; FARIA FILHO, D.E.; OELKE, C.A. Efeito da idade da matriz no tempo de eclosão, tempo de Permanência do neonato no nascedouro e o peso do pintainho. Archives of Veterinary Science, v.11, p.45-49, 2006. 
Rev. Bras. Saúde Prod. Anim., Salvador, v.17, n.2, p.214-221 abr./jun., 2016 http://www.rbspa.ufba.br ISSN 15199940

AVILA, V.S.; PENZ JUNIOR, A.M.; BRUM, A.R. de; GUIDONI, A.L.; ROSA, P.S.; COLDEBELLA, A. Produção e qualidade de ovos em reprodutoras de frangos de corte com horário de arraçoamento diferenciado. Revista Brasileira de Zootecnia, v.34, p.1202-1209, 2005.

BARBOSA, V.M.; CANÇADO, S.V.; BAIÃO, N.C.; LANA, A.M.O.; LARA, L.J.C.; SOUZA, M.R. Efeitos da umidade relativa do ar na incubadora e da idade da matriz leve sobre o rendimento da incubação. Arquivo Brasileiro de Medicina Veterinária e Zootecnia, v.60, p.741-748, 2008.

CARVALHO, F.B.; STRIGHINI, J.H.; JARDIM FILHO, R.M.; LEANDRO, N.S.M.; CAFÉ, M.B.; DE DEUS, H.A.S.B. Qualidade interna e da casca para ovos de poedeiras comerciais de diferentes linhagens e idades. Ciência Animal Brasileira, v.8, p.25-29, 2007.

CASTELLÓ, J.A.L.; PONTES, M.; GONZÁLEZ, F.F. Producción de huevos. 1.ed. Barcelona: Real Escuela de Avicultura, 1989. 367p.

FÁVERO, P.F. O manejo como melhoria na fertilidade de galos e galinhas.

Avicultura Industrial, v.102, p.29-35, 2010.

FEREIRA, D.F. SISVAR - Sistema de análises de variância. Versão 5.3. Lavras, MG: UFLA, 2010.

INGRAM, D.R.; HATTEN, L.F.; HOMAN, K.D. A study on the relationship between eggshell color and eggshell quality in commercial broiler breeders. International Journal of Poultry Science, v.7, p.700-703, 2008.
MOYLE, J.; YOHO, D.; BRAMWELL, $\mathrm{K}$. Measuring hatching egg shell quality. Avian advice, v.10, p.7-9, 2008.

PEEBLES, E. D.; BRAKE, J.

Relationship of eggshell porosity to stage of embryonic development in broiler breeders. Poultry Science, v.64, p.23882391, 1985.

ROCHA, J. S. R.; LARA, L. J. C.; BAIÃO, N. C.; CANÇADO, S. V.; TRIGINELLI, M. V.; LEITE, J. F. C. Efeito da classificação dos ovos sobre a uniformidade, o desempenho e o rendimento de abate de frangos de corte.

Arquivo Brasileiro de Medicina Veterinária, v.60, nº5, p.1181-1187, 2008.

ROSA, P.S.; AVILA, V.S. Variáveis relacionadas ao rendimento da incubação de ovos em matrizes de frangos de corte. Concórdia, SC: Embrapa Suínos e Aves, 2000. p.1-3. (Comunicado Técnico, 246).

ROSA, P.S.; GUIDONI, A.L.; LIMA, I.L.; BERSCH, F.X.R. Influência da temperatura de incubação em ovos de matrizes de corte com diferentes idades e classificados por peso sobre os resultados de incubação. Revista Brasileira de Zootecnia, v.31, p.1011-1016, 2002.

ROQUE, L.; SOARES, M.C. Effects of Eggshell Quality and Broiler Breeder Age on Hatchability. International Journal of Poultry Science, v.73, p.1838-1845, 1994.

SANTOS, J.E.C. dos; GOMES, F.S.; BORGES, G.L.F.N.; SILVA, P.L.; CAMPOS, E.J.; FERNADES, E.A.; GUIMARÃES, E.C. Efeito da linhagem e da idade das matrizes na perda de peso dos ovos e no peso embrionário durante a incubação artificial. Bioscience Journal, v.25, p.163-169, 2009. 
Rev. Bras. Saúde Prod. Anim., Salvador, v.17, n.2, p.214-221 abr./jun., 2016 http://www.rbspa.ufba.br ISSN 15199940

TANURE, C.B.G.S.; CAFÉ, M.B.;

LEANDRO, N.S.M.; BAIÃO, N.C.;

STRIGHINI, J.H.; GOMES, N.A.

Efeitos da idade da matriz leve e do

período de armazenamento de ovos

incubáveis no rendimento de incubação.

Arquivo Brasileiro de Medicina

Veterinária Zootecnia, v.61, p.1391-

1396, 2009.

Data de recebimento: 04/02/2015

Data de aprovação: 15/04/2016 